\title{
Efficacy of Nutrition Screening Tools in Predicting the Length of Hospital Stay in Older Hospitalized Patients
}

Cafer Balcı, [MD]

ORCID: 0000-0002-1478-1106

\section{ree) ABSTRACT Cere}

Objective: The prevalence of malnutrition remains high in older hospitalized patients. Subjective Global Assessment, the Nutrition Risk Screening-2002, and Malnutrition Universal Screening Tool are widely used screening and assessment tools, but comparison of their efficacy in predicting clinical outcomes like length of hospital stay remain scarce. This study aimed to compare the efficacy of these tools in predicting length of hospital stay in a group of older hospitalized patients.

Materials and Methods: A retrospective analysis was performed in a sample of 72 patients consecutively admitted to a geriatric medicine ward. Subjective Global Assessment, Nutrition Risk Screening-2002 and Malnutrition Universal Screening Tool were performed within 24 hours of admission. Patients were classified as having prolonged length of hospital stay if they stay in the hospital for more than ten days. The association of baseline malnutrition defined by each tool and the prolonged length of hospital stay was assessed using unadjusted and adjusted logistic regression models.

Results: The mean age of the patients was $73.5 \pm 6.9$ years, and $61.1 \%$ were women. The prevalence of malnutrition was $45.8 \%$ with Subjective Global Assessment, 51.4\% with Nutrition Risk Screening-2002, and 33.3\% with Malnutrition Universal Screening Tool. Among the entire cohort, twenty-nine patients (40.2\%) had longer length of the hospital stay. After adjusted for covariates, multivariate logistic regression analysis revealed that the Subjective Global Assessment had the best predictive power (OR: 3.9; $p$ : 0.02), followed by Nutrition Risk Screening-2002 (OR: 3.8; $p: 0.03$ ), and Malnutrition Universal Screening Tool (OR: 2.9; $p: 0.02$ ).

Conclusion: Malnutrition assessed by the Subjective Global Assessment, Nutrition Risk Screening-2002 and Malnutrition Universal Screening Tool on admission predict prolonged length of hospital stay in hospitalized older patients.

Keywords: Nutrition status screening, length of hospital stay, older patient

https://doi.org/10.32552/2021.ActaMedica.535

Received: 8 November 2020, Accepted: 12 January 2021,

Published online: 2 February 2021

\section{INTRODUCTION}

Disease-related malnutrition has been shown to be associated with higher morbidity and mortality, prolonged recovery from illness and length of hospital stay (LOS) [1-3]. Moreover, nutrition status of the patients may deteriorate during hospital stay [2]. Adaptation of the older patients to disease related metabolic stress is poor [4], and advanced age is recognized as an independent predictor of nutrition status derangements and worse clinical outcome [5]. However, malnutrition on admission is often unrecognized and undertreated in about two thirds of cases, including older patients $[6,7]$. 
Nutrition screening and assessment tools can assist in the early identification of the presence of malnutrition and initiation of prompt intervention to improve the nutrition status of the older hospitalized patients. There are numerous screening and assessment tools for nutrition status assessment [8]. In the absence of a universally recognized 'gold standard' for malnutrition [9], their comparison is of particular interest. True validity of a screening or assessment tool can only be discussed when its impact on clinical outcome has been proven [9]. LOS is a relevant outcome parameter in terms of morbidity and hospital cost [10]. Little information is available regarding the comparability of three commonly used nutrition tools, Subjective Global Assessment (SGA) [11], Nutritional Risk Screening Instrument (NRS-2002) [12], and Malnutrition Universal Screening Tool (MUST) [13] in older hospitalized patients. Therefore, the aim of this study was compare the association between nutrition status at hospital admission, assessed by SGA, NRS-2002 and MUST with time to discharge in older hospitalized patients.

\section{MATERIAL and METHODS}

\section{Study Design and Sample}

This was a retrospective cross-sectional cohort study. Patients aged 65 years and over who were admitted to a geriatric medicine ward during a 7-month period were included in the study. Patients were excluded from the study if classified as palliative, with an estimated length of stay less than 24 hours or could not weighted because of poor medical condition. A total of 72 patients who were eligible for the study were included. This study has been approved by the ethics committee and conducted in accordance with the Declaration of Helsinki (2000). Informed consent was waived because of the retrospective design of the study.

\section{Data Collection and Variable Definition}

All recruited patients were assessed within 24 hours of admission by a single geriatrician. Data on patients' demographic characteristics, medical history including comorbidities and medications currently taken, anthropometric measurements including body weight, height, body mass index (BMI), mid-upper calf circumference (MUAC), calf circumference (CC) and hand grip strength
(HGS), and laboratory test including albumin were recorded. Anthropometric measurements including weight, height, MUAC, CC, and hand grip strength were performed according to the standardized and recommended procedures and techniques [12]. The patients weighed in light clothing and without shoes, using a floor calibrated scale. Height was measured barefoot while the patient was standing in upright position, standing against a wall and looking forward by the aid of a tape measure, and was recorded in centimeters. BMI was calculated according to the equation: $\mathrm{BMI}=$ weight $(\mathrm{kg}) /$ height $^{2}(\mathrm{~m})$. Calf circumference was measured twice while the patient is sitting, pressing the foot completely on the floor, flexing the knee 90 degrees by using a measuring cylinder from the largest portion of the calf. Care was taken not to compress subcutaneous tissue. The arithmetic mean was recorded in centimeters with a sensitivity of $0.1 \mathrm{~cm}$. Hand grip strength was measured with a digital dynamometer (TKK 5401 Grip-D; Takei, Niigata, Japan) and each device was calibrated prior to the initiation of data collection. Patients performed the test sitting on a bed or chair, while their shoulder adducted and neutrally rotated, elbow flexed at $90^{\circ}$ and wrist neutrally positioned. The patient's dominant hand was used for the assessment. Each patient was given a demonstration before the measurement and then asked to complete a total of three maximal isometric contractions. The average readings showing on the display of the dynamometer were recorded, and the mean hand grip strength was calculated.

The nutrition status of the patients were evaluated by using the Subjective Global Assessment (SGA), Nutrition Risk Screening 2002 (NRS-2002) and Malnutrition Universal Screening Tool (MUST).

SGA is a semi quantitative tool to assess the nutrition status based on the patient's history including weight loss, change in dietary intake, digestive organ symptom, physical function, and underlying disease and on physical findings such as subcutaneous fat, quantity of skeletal muscle, presence of edema, and ascites [11]. Patients with severe malnutrition were classified as $C$, moderate malnutrition as $\mathrm{B}$, and normal nutrition as $\mathrm{A}$.

NRS-2002 consists of 4 questions as an initial screening including $\mathrm{BMI}$, weight loss, reduced dietary intake, and severe illness [12]. If at least 1 of these questions is answered as yes, the final 
screening part consisting of nutrition parameters and grading of severity of disease is performed. The nutrition status of all patients was evaluated with a scoring system. The patients were considered "nutritionally at risk" when the total NRS-2002 score was $\geq 3$.

MUST is a valid and reliable tool that uses Body Mass Index (BMI), recent weight change and the effects of acute disease on nutritional intake to give an overall score for risk of malnutrition [13]. If the total score is zero, the patient is at low risk of malnutrition, a score of 1 indicates medium risk and if the score is 2 or greater, the patient is at high risk of malnutrition.

Nutrition status of the patients was assessed within 24 hours of admission. Patients with SGA class $B$ and C, those with a NRS-2002 score of $\geq 3$, and those with a MUST score of $\geq 1$ were considered as malnourished for the analysis.

Length of hospital stay (LOS) was the primary outcome. It was determined as the number of days of hospitalization, including the date of admission and excluding that of discharge. Those who were discharged within 24 hours were not included in the study. The admission and discharge of each patient was decided by the geriatrician solely according to medical judgment to avoid the possible effect of patients' convenience on the length of hospital stay.

\section{Statistical Analysis}

SPSS for Windows v.23.0 (SPSS Inc, Chicago, IL) was used for the statistical analyses. Variables were examined using visual (histograms and probability plots) and analytical methods to determine whether they were normally distributed. Categorical variables were shown as numbers and frequencies, with differences being analyzed by the $x 2$ test or Fisher exact test, where appropriate. Continuous data that followed a normal distribution was described with mean and SD, and between-group comparisons were performed by independent samples t-test. When distributions were not normal, the data were described with median (minmax) and group comparisons were done using the Mann-Whitney $U$ test. LOS was dichotomized as those hospitalized for $>10$ days vs $\leq 10$ days. The area under curve (AUC) values were calculated based on receiver operating characteristics (ROC) curves analysis to assess the predictive accuracy of the SGA, NRS-2002 and the MUST regarding LOS. Multivariable logistic regression analyses were performed to examine the relationship between the prolonged length of hospital stay and the presence of malnutrition according to the SGA, NRS-2002 and the MUST. Unadjusted analyses were done in Model 1. In Model 2, the results were adjusted for age, gender, Charlson Comorbidity Index and the cause of hospitalization. The results were expressed as odds ratios (ORs) and corresponding 95\% Cls. Hosmer-Lemeshow goodness-of-fit statistics were used to assess model fit. All reported $P$-values were based on 2-sided tests and compared to a significance level of $5 \%$.

\section{RESULTS}

A total of 72 patients were included in the study, of whom $44(61.1 \%)$ were women. Mean age was $73.5 \pm 6.9$ years, ranging from 65 to 88 years. Table 1 shows the patients' baseline characteristics and the comparison between duration of LOS. There was no difference in the age and gender distribution among groups. The prevalence of malnutrition among all patients at admission was $45.8 \%$ based on SGA, 51.4\% using NRS-2002, and 33.3\% according to the MUST. Among the entire cohort, twenty-nine patients (40.2\%) had longer length of the hospital stay (LOS). Patients who were classified as having prolonged LOS had higher prevalence of malnutrition based on either SGA $(62.1 \%$ vs $31.9 \%$; $p: 0.003), \mathrm{NRS}-2002$ (65.5\% vs $41.9 \% ; p: 0.04)$, and MUST (48.3\% vs $23.3 \%$; $p$ : 0.04$)$ than that of nonprolonged LOS ( $\leq 10$ days) (Table 1$)$. Furthermore, patients who had prolonged LOS had lower weight, calf circumference, hand grip strength, and serum albumin levels than that of non-prolonged LOS (Table 1). The Charlson Comorbidity Index scores, the distribution of chronic diseases, and the cause of hospitalization were comparable in each group (Table 1).

The AUC values based on ROC analysis to assess the predictive accuracy of each tool regarding the prolonged LOS are shown in Table 2. According to the ROC analysis, the largest AUC was observed in the SGA (AUC, 0.636; $95 \% \mathrm{Cl}, 0.504-0.768 ; p$ : 0.048), followed by the NRS-2002 (AUC, $0.618 ; 95 \% \mathrm{Cl}, 0.486$ - 0.751; $p$ : 0.049). The lowest AUC was observed in the MUST (AUC, $0.605 ; 95 \% \mathrm{Cl}, 0.461-0.740 ; p: 0.05$ ). 
Table 1. Baseline characteristics and comorbidities of the patients and the comparison between the categories of length of stay

\begin{tabular}{|c|c|c|c|c|}
\hline & $\begin{array}{l}\text { Total } \\
(\mathrm{n}: 72)\end{array}$ & $\begin{array}{l}\text { Prolonged LOS } \\
\qquad(\mathrm{n}: 29)\end{array}$ & $\begin{array}{l}\text { Non-prolonged LOS } \\
\qquad(\mathrm{n}: 43)\end{array}$ & $\begin{array}{c}p \\
\text { value }\end{array}$ \\
\hline Age, y & $73.5 \pm 6.9$ & $73.2 \pm 5.7$ & $73.7 \pm 7.7$ & 0.85 \\
\hline Women, n (\%) & $44(61.1 \%)$ & $16(55.2 \%)$ & $28(65.1 \%)$ & 0.46 \\
\hline Height, cm & $157.6 \pm 9.9$ & $157.2 \pm 9.8$ & $157.9 \pm 10.1$ & 0.87 \\
\hline Weight, kg & $70.4 \pm 14.7$ & $67.7 \pm 13.2$ & $72.3 \pm 15.5$ & 0.04 \\
\hline Body mass index & $28.3 \pm 6.2$ & $27.6 \pm 5.6$ & $28.8 \pm 6.5$ & 0.5 \\
\hline Calf circumference, cm & $33.9 \pm 4.4$ & $32.7 \pm 4.5$ & $34.7 \pm 4.2$ & 0.05 \\
\hline Hand grip strength, kg & $19.4 \pm 8.1$ & $17.4 \pm 7.8$ & $20.9 \pm 8.1$ & 0.04 \\
\hline Serum albumin level, mg/dL & $3.6 \pm 0.5$ & $3.4 \pm 0.5$ & $3.8 \pm 0.4$ & 0.002 \\
\hline Charlson Comorbidity Index, median (min-max) & $5(0-11)$ & $5(0-11)$ & $5(0-9)$ & 0.77 \\
\hline \multicolumn{5}{|l|}{ Comorbidities, n (\%) } \\
\hline Hypertension & $49(68.1 \%)$ & $19(65.5 \%)$ & $30(69.8 \%)$ & 0.79 \\
\hline Diabetes mellitus & $26(36.1 \%)$ & $8(27.6 \%)$ & $18(41.9 \%)$ & 0.31 \\
\hline Coronary artery disease & $22(30.6 \%)$ & $8(27.6 \%)$ & $14(32.6 \%)$ & 0.79 \\
\hline Chronic kidney disease & $22(30.6 \%)$ & $8(27.6 \%)$ & $14(32.6 \%)$ & 0.79 \\
\hline Dementia & $8(11.1 \%)$ & $4(13.8 \%)$ & $4(9.3 \%)$ & 0.71 \\
\hline \multicolumn{5}{|l|}{ Causes of hospitalization, n (\%) } \\
\hline Infectious & $25(34.7 \%)$ & $10(34.5 \%)$ & $15(34.9 \%)$ & \\
\hline Non-infectious & $47(65.3 \%)$ & $19(65.5 \%)$ & $28(65.1 \%)$ & 0.97 \\
\hline Total medication number, median (min-max) & $4(0-11)$ & $4(0-11)$ & $4(0-10)$ & 0.31 \\
\hline \multicolumn{5}{|l|}{ Malnutrition, n (\%) } \\
\hline SGA class B and C & $33(45.8 \%)$ & $18(62.1 \%)$ & $15(34.9 \%)$ & 0.03 \\
\hline NRS-2002 score $\geq 3$ & 37 (51.4\%) & $19(65.5 \%)$ & $18(41.9 \%)$ & 0.04 \\
\hline MUST score $\geq 1$ & $24(33.3 \%)$ & $14(48.3 \%)$ & $10(23.3 \%)$ & 0.04 \\
\hline
\end{tabular}

LOS, Length of Hospital Stay; SGA, Subjective Global Assessment; NRS-2002, Nutrition Risk Screening Tool -2002; MUST, Malnutrition Universal Screening Tool

Table 2. Area under ROC curve values of the three nutrition screening/assessment tools according to the prolonged length of hospital stay outcome

\begin{tabular}{|l|c|c|}
\hline Nutrition tools & Area under ROC curve $[95 \% \mathrm{Cl}]$ & $p$ value \\
\hline SGA & $0.636(0.504-0.768)$ & 0.048 \\
NRS-2002 & $0.618(0.486-0.751)$ & 0.049 \\
MUST & $0.605(0.461-0.740)$ & 0.05 \\
\hline
\end{tabular}

ROC, Receiver Operating Characteristics; SGA, Subjective Global Assessment; NRS-2002, Nutrition Risk Screening Tool -2002; MUST, Malnutrition Universal Screening Tool

The unadjusted and the adjusted logistic regression analyses are shown in Table 3. In the unadjusted Model 1, malnutrition defined by either each nutrition tool was significantly associated with prolonged LOS (SGA OR, 4.5 [ $p$ : 0.025]; NRS-2002 OR, 4.3 [p: 0.034]; MUST OR, 3.8 [p: 0.028]) compared with well-nourished older patients. The association of malnutrition with prolonged LOS was maintained when age, gender, Charlson Comorbidity Index, and the cause of hospitalization were added as covariates in the adjusted Model 2 (Table 3).

\section{DISCUSSION}

The average population age is increasing in worldwide, causing a rise in older adults with consequently greater need of hospitalization [14]. Although widely underdiagnosed, the prevalence of malnutrition in hospitalized older patients remains excessively high, reaching 30-55\%, depending on the patient population, settings and tools used [15]. It is well established that, timely nutrition treatment, especially on admission, is beneficial in 
Table 3. Odds Ratio of Prolonged Hospital Stay According to Nutrition Status by Different Nutrition Tools

\begin{tabular}{|c|c|c|c|c|}
\hline & \multicolumn{2}{|c|}{ Model 1} & \multicolumn{2}{|c|}{ Model $2^{*}$} \\
\hline & OR [95 \%Cl] & $p$ value & OR [95 \%Cl] & $p$ value \\
\hline \multicolumn{5}{|l|}{ SGA } \\
\hline Class A & 1 & - & 1 & - \\
\hline Class $B$ and $C$ & 4.5 (1.9-13.9) & 0.025 & $3.9(1.2-12.2)$ & 0.02 \\
\hline \multicolumn{5}{|l|}{ NRS-2002 score } \\
\hline$<3$ & 1 & - & 1 & - \\
\hline$\geq 3$ & $4.3(1.8-14.8)$ & 0.034 & $3.8(1.1-13.1)$ & 0.03 \\
\hline \multicolumn{5}{|l|}{ MUST score } \\
\hline$<1$ & 1 & - & 1 & - \\
\hline$\geq 1$ & $3.8(1.4-11.2)$ & 0.028 & $2.9(1.2-9.9)$ & 0.02 \\
\hline
\end{tabular}

SGA, Subjective Global Assessment; NRS-2002, Nutrition Risk Screening Tool -2002; MUST, Malnutrition Universal Screening Tool

* Adjusted for age, gender, Charlson Comorbidity Index, and the cause of hospitalization

reducing complication rates of malnutrition $[16,17]$. Even though routine assessment of nutrition status of patients in hospitalized older adults is recommended, there is little consensus as to which tool should be used [8].

This study aimed to compare three different nutrition tools, SGA, NRS-2002 and MUST, in order to evaluate the nutrition status of hospitalized older patients at admission, and their predictive ability of prolonged length of hospital stay. In the present study, malnutrition, according to the NRS-2002, was found in $51.4 \%$ of the patients, whereas the prevalence of malnutrition was $45.8 \%$ according to the SGA and $33.3 \%$ according to the MUST. Although the prevalence of malnutrition defined by SGA was lower than malnutrition based on NRS2002, the logistic regression analyses revealed that the SGA was better than NRS-2002 and MUST at predicting prolonged LOS. SGA focuses more on chronic or established nutrition risk rather than acute nutritional changes compared to NRS-2002, which may explain this discrepancy.

When compared to SGA and NRS-2002, the ability of MUST was lower in predicting prolonged LOS. Notably, the MUST systematically classifies patients with an acute condition as being at high nutritional risk, whereas chronic conditions are not classified according to their severity. As a result, this tool tends to overestimate high nutritional risk and underestimate intermediate nutritional risk. This could be responsible for the different capacity of MUST for predicting prolonged LOS when compared to SGA and NRS-2002.
The association between poor nutrition status defined by different nutrition screening and assessment tools and LOS is well established in different settings $[2,10,18-20]$. The results of present study are in line with the existing literature, furthermore provide additional evidence regarding the predictive validity of SGA, NRS-2002 and MUST tools with regard to prolonged LOS in older hospitalized patients.

However, in a study examining the association of the nutrition status at admission defined by NRS2002, Mini Nutritional Assessment (MNA), NRS2002, SGA, and Malnutrition Screening Tool (MST) and LOS in older hospitalized patients, the authors found that NRS-2002 (crude OR, 3.01; $p<0.002$ ), SGA (crude OR, 2.67; $p<0.005$ ), and MNA (crude OR, $2.53 ; p<0.005)$ were associated with prolonged LOS in the unadjusted logistic regression analyses [21]. However, only NRS-2002 (OR, 2.25; $p$ :0.04) was found to be an independent risk factor for prolonged LOS in the adjusted regression analyses. SGA and MNA were not statistically significant when adjusted for gender, education, interviewer and the severity of illness according to the regression analyses. Unlike this present study, patients admitted to orthopedictrauma wards were included in that study, which do not represent the full spectrum of nutritionally relevant pathologies. This could explain the loss of significant association between nutrition tools (SGA and MNA) and LOS in the adjusted regression models. Considering all these, further studies evaluating the validity of nutrition tools in different settings among comparable populations are needed. 
Length of hospital stay may reflect the prognosis of the older patient and has been frequently used as an outcome [22]. Moreover, predicting the length of stay has proven to be an important mission to support the establishment of an adequate health care plan by the medical team and for an efficient management of the limited health care resources [23]. Several factors are determinants of hospital length of stay, patient-related factors such as age, cause of hospitalization, and comorbidities [24]. In this study cohort, these confounding factors were distributed equally among the prolonged and non-prolonged LOS groups. Furthermore, the association of malnutrition defined by each nutrition tool and the prolonged LOS was maintained in the present study after controlling for age, gender, comorbidities, and the cause of hospitalization in the adjusted regression model.

Some limitations of the present study need to be kept in mind while interpreting the data. First, this was a retrospective analysis of data collected in a single center. The generalizability of the findings may be limited because of the study population chosen and the study group included. The study population consisted of only older hospitalized patients in geriatric medicine ward, and therefore, the results of this study may not be generalizable to other services like intensive care units or community-dwelling populations. The present study lacked information on severity of diseases, and functional status that should be included in studies of predictors of clinical outcome. Finally, nutrition intervention and the treatment of the cause of hospitalization during hospital stay were not recorded and they could have shortened patients' hospitalization introducing a differential bias in malnourished and well-nourished groups.

In conclusion, the results of this study show that, SGA, NRS-2002 and MUST have predictive validity in the older hospitalized population with regard to prolonged LOS. When compared to NRS-2002 and MUST, SGA is more useful in predicting LOS. The findings of this study support the recommendation to screen patients for the presence of malnutrition in older inpatients so that an appropriate treatment plan can be implemented, with a view to improving clinical outcome. This study provides evidence that nutrition screening and assessment tools can detect patients at risk of adverse outcome like prolonged length of hospital stay in this vulnerable population regardless of the instrument used. Further studies are needed determine the cost-effectiveness of interventions based on nutrition scoring methods.

\section{ACKNOWLEDGEMENT}

The author would like to thank all nurses of the geriatric medicine ward.

\section{CONFLICT Of INTEREST STATEMENT}

The author has no conflict of interest to declare. 
[1] Norman K, Pichard C, Lochs H, et al. Prognostic impact of disease-related malnutrition. Clinical nutrition 2008; 27:515.

[2] Caccialanza R, Klersy C, Cereda E, et al. Nutritional parameters associated with prolonged hospital stay among ambulatory adult patients. Cmaj 2010; 182:18431849.

[3] Caccialanza R, Cereda E, Klersy C. Malnutrition, age and inhospital mortality. Cmaj 2011; 183:826-826.

[4] Schneider SM, Al-Jaouni R, Pivot X, et al. Lack of adaptation to severe malnutrition in elderly patients. Clinical Nutrition 2002; 21:499-504.

[5] Sorensen J, Kondrup J, Prokopowicz J, et al. EuroOOPS: an international, multicentre study to implement nutritional risk screening and evaluate clinical outcome. Clinical nutrition 2008; 27:340-349.

[6] Kelly I, Tessier S, Cahill A, et al. Still hungry in hospital: identifying malnutrition in acute hospital admissions. Qjm 2000; 93:93-98.

[7] Mowé $M, B ø h m e r ~ T$. The prevalence of undiagnosed protein-calorie undernutrition in a population of hospitalized elderly patients. Journal of the American Geriatrics Society 1991; 39:1089-1092.

[8] Cederholm T, Barazzoni R, Austin P, et al. ESPEN guidelines on definitions and terminology of clinical nutrition. Clinical nutrition 2017; 36:49-64.

[9] Guaitoli PR, Jansma EP, de Vet HC. Nutrition screening tools: does one size fit all? A systematic review of screening tools for the hospital setting. Clinical nutrition 2014; 33:39-58.

[10] Kyle ÚG, Kossovsky MP, Karsegard VL, et al. Comparison of tools for nutritional assessment and screening at hospital admission: a population study. Clinical Nutrition 2006; 25:409-417.

[11] Detsky AS, Baker J, Johnston N, et al. What is subjective global assessment of nutritional status? Journal of parenteral and enteral nutrition 1987; 11:8-13.

[12] Bolayir B, Arik G, Yeşil Y, et al. Validation of Nutritional Risk Screening-2002 in a Hospitalized Adult Population. Nutrition in Clinical Practice 2019; 34:297-303.

[13] Elia M. Screening for malnutrition: a multidisciplinary responsibility. Development and use of the Malnutrition
Universal Screening Tool ('MUST') for adults Redditch: BAPEN 2003.

[14] Wittenberg R, Sharpin L, McCormick B, et al. The ageing society and emergency hospital admissions. Health Policy 2017; 121:923-928.

[15] Covinsky KE, Martin GE, Beyth RJ, et al. The relationship between clinical assessments of nutritional status and adverse outcomes in older hospitalized medical patients. Journal of the American Geriatrics Society 1999; 47:532538.

[16] Somanchi M, Tao X, Mullin GE. The facilitated early enteral and dietary management effectiveness trial in hospitalized patients with malnutrition. Journal of Parenteral and Enteral Nutrition 2011; 35:209-216.

[17] Holyday M, Daniells S, Bare M, et al. Malnutrition screening and early nutrition intervention in hospitalised patients in acute aged care: a randomised controlled trial. The journal of nutrition, health \& aging 2012; 16:562-568.

[18] Mendes J, Alves P, Amaral T. Comparison of nutritional status assessment parameters in predicting length of hospital stay in cancer patients. Clinical nutrition 2014; 33:466-470.

[19] Borek P, Chmielewski M, Małgorzewicz S, et al. Analysis of outcomes of the NRS 2002 in patients hospitalized in nephrology wards. Nutrients 2017; 9:287.

[20] Wang F, Chen W, Bruening KS, et al. Nutrition screening tools and the prediction of clinical outcomes among chinese hospitalized gastrointestinal disease patients. PloS one 2016; $11: \mathrm{e} 0159436$.

[21] Martins C, Correia JR, do Amaral TF. Undernutrition risk screening and length of stay of hospitalized elderly. Journal of Nutrition for the Elderly 2006; 25:5-21.

[22] Bohannon RW. Hand-grip dynamometry predicts future outcomes in aging adults. Journal of geriatric physical therapy 2008; 31:3-10.

[23] Amaral TF, Matos LC, Tavares MM, et al. The economic impact of disease-related malnutrition at hospital admission. Clinical nutrition 2007; 26:778-784.

[24] Mozes B, Schiff E, Modan B. Factors affecting inappropriate hospital stay. International Journal for Quality in Health Care 1991; 3:211-217. 